\title{
Compatibility of organic solvents for electrochemical measurements in PDMS-based microfluidic devices
}

\author{
W. Adamiak ${ }^{1}$ D. Kałuża ${ }^{1}$ - M. Jönsson-Niedziolka ${ }^{1}$ (I)
}

Received: 4 May 2016 / Accepted: 10 August 2016 / Published online: 20 August 2016

(C) The Author(s) 2016. This article is published with open access at Springerlink.com

\begin{abstract}
We have investigated the compatibility of some organic solvents commonly used in electrochemistry with microfluidic channels based on poly(dimethylsiloxane) (PDMS) and compared the stability of electrochemical measurements over several hours with how much PDMS swells when immersed in these solvents. Lee et al. (Anal Chem 75: 6544-6554. doi:10.1021/ac0346712, 2003) have shown that there is a good correlation between swelling of PDMS and the solubility parameter $\left(\delta_{\mathrm{H}}\right)$ of the various solvents and suggested that $\delta_{\mathrm{H}}$ can function as an indication of PDMS compatibility. We show that solvents with a very high swelling ratio can give stable voltammetry over several hours, and thus, we do not find that swelling is a good measure for compatibility with PDMS in electrochemical experiments.
\end{abstract}

Keywords Microfluidics · PDMS · Organic solvents · Swelling $\cdot$ Solubility parameter $\cdot$ Electrochemistry

\section{Introduction}

Microfluidic devices can be fabricated from different materials like silicon (Reyes et al. 2002), glass, (Whitesides 2002) hydrogel, (Ghaemmaghami et al. 2012) paper, (Cate et al. 2015) or polymers (Ren et al. 2013). Each of them

Electronic supplementary material The online version of this article (doi:10.1007/s10404-016-1792-4) contains supplementary material, which is available to authorized users.

M. Jönsson-Niedziolka

martinj@ichf.edu.pl

1 Institute of Physical Chemistry, Polish Academy of Sciences, Kasprzaka 44/52, 01-224 Warsaw, Poland possesses their drawbacks and advantages. Historically, the first materials for microfluidic device fabrication were glass and silicon, and the chips were made by wet etching and then fusion bonding procedures. Because of the stiffness and chemical resistivity of these materials, processing usually requires the use of dangerous chemicals like hydrofluoric acid (in the wet etching step) and super clean environments together with high temperature and pressure conditions (in the fusion bonding step). These factors increase the overall cost of microfluidic chips fabrication and limit the broader use of these materials.

Over the last few years, devices made from paper where the liquid solutions are moved along the chip by capillary forces have become increasingly popular (Martinez et al. 2007). Since the channels are open to air and there is a cellulose matrix in the channel area, the number of analytical methods that can be integrated with these devices is also limited. So far, the most commonly used method is colorimetry, whose application to paper chips seems to be the main strategy for their commercial applications, mainly due to minimum requirements of the equipment needed for analysis (Martinez et al. 2008). Another drawback of the paper chips is that they cannot be integrated with small size valves which are used for precise control and manipulation of the flowing liquids.

For laboratory research-where one needs to compromise the ease of fabrication and the performance of the device-the most commonly used materials are elastomers, and among these, poly(dimethylsiloxane) (PDMS) is the most popular one (McDonald and Whitesides 2002). The main advantage of PDMS is the convenience in fabrication-it can be cast with high resolution in a relatively simple and fast photolithography process (Wu et al. 2003). Due to PDMS elasticity, it is possible to fabricate microvalves which can act like on-demand filters (Unger et al. 2000). 
Additionally, PDMS is gas permeable which makes it suitable for long-term cell culture in biological studies like cell screening (Kim et al. 2015), biochemical assays (El-Ali et al. 2006) and single cell analysis (Mu et al. 2013). On the other hand, permeability to gases can cause water evaporation during the flow and simultaneous crystallization of the dissolved analytes. This apparent disadvantage has been already used to perform controlled protein crystallization (Shim et al. 2007).

For integration of PDMS microfluidic channels with electrochemical experiments, PDMS structures are usually bound to a glass substrate containing the electrodes. In this way, the electrochemical microfluidics has been used to lower detection limits in electrochemical sensing devices (Pumera et al. 2006; Swensen et al. 2009), to improve the performance of biofuel cells (Kjeang et al. 2009) and in fundamental research like determination of electrocatalytic (Dumitrescu et al. 2012) or enzymatic reaction kinetics, (Han et al. 2009) investigation of molecular interactions (Strutwolf et al. 2010) or studying electrochemically modulated charge transfer processes at soft interfaces between two co-flowing liquid phases (MacDonald et al. 2007; Kaluza et al. 2013, 2014).

In most cases, however, electrochemical applications of PDMS microfluidic systems have been limited to the use of aqueous solutions or a few examples of aqueous-organic two-phase flows, with no reports on the use of organic solvents only. The use of organic solvents in microfluidics is an important issue that would expand the use of PDMS microfluidics over such fields as electrosynthesis, electroanalysis of oil samples or electrochemical extraction. It can also lead to an increase in the output of the two-phase chemical processes (like hydrogen peroxide generation) (Jedraszko et al. 2013) by transferring them from static to microfluidic conditions, as has been already shown for electrosynthesis of drug metabolites (Stalder and Roth 2013). In terms of application of electrochemical techniques in lab-on-a-chip detection units, equally important is the time scale of the analysis, i.e. how long one can perform reliable measurements in the non-aqueous sample without significant damage of the microfluidic device. So far, compatibility of PDMS with typical organic solvents has been addressed by Whitesides and co-workers (Lee et al. 2003), who studied physical effects like swelling of PDMS or its dissolution in more than 30 different solvents. In their studies, however, no effect of the organic solvents on the electrochemical measurements has been discussed.

The aim of this work was to examine how much a selection of organic solvents, commonly used in electrochemical measurements, affects PDMS physical properties and if there is any effect of so-called PDMS non-compatible solvents on the recorded electrochemical signals. Earlier, Whitesides and co-workers found that non-compatible organic solvents either swell or dissolve PDMS (Lee et al. 2003). To quantify the swelling effect, they introduced a swelling parameter which simply describes how much the linear dimension of PDMS change when it is immersed in a given solvent. In this work, we have performed electrochemical measurements in six organic solvents commonly used in electrochemical studies; 1,2-dichlorobenzene (DCB) (Kowski et al. 2009); 4-(3-phenylpropyl)pyridine (PPP) (Katif et al. 2008); $\mathrm{N}$-octyl-2-pyrollidone (NOP) (MacDonald et al. 2007; Kaluza et al. 2013, 2014); $\alpha, \alpha, \alpha$-trifluorotoluene (TFT) (Olaya et al. 2012); toluene (Duffy and Bond 2006; Adamiak et al. 2011); and acetonitrile (ACN) (MacDonald et al. 2008). For four of these solvents (DCB, PPP, NOP, TFT) that were not included in Whitesides' study, we also determined the swelling parameter. In the case of TFT, we have used confocal fluorescence microscopy to visualise the real-time penetration of the solvent through PDMS walls. We believe that the conclusions from this work can expand the use of PDMS microfluidics in non-aqueous solvents in general, and the use of electrochemical instrumentation in these environments in particular.

\section{Experimental}

\subsection{Chemicals and materials}

The poly(dimethylsiloxane) (PDMS) used for all experiments was Sylgard 184 elastomer and curing agent obtained from Dow Corning Company. We prepared the PDMS by mixing a 10:1 ratio (by weight) elastomer with curing agent which was degassed in a vacuum desiccator and cured at $70{ }^{\circ} \mathrm{C}$ for $24 \mathrm{~h}$. The channels $(500 \mu \mathrm{m} \times 90 \mu \mathrm{m} \times 19 \mathrm{~mm}$, whl), with a single inlet and outlet, were prepared by soft lithography as previously described (Kaluza et al. 2013). The samples for the swelling study were cuboids of size approximately $1 \times 1 \times 0.5 \mathrm{~cm}$. The electrochemical experiments were performed using ferrocene ( $99 \%$, Alfa-Aesar) as a redox probe and tetrabutylammonium-perchlorate (TBAP, $99 \%$, Fluka) as supporting electrolyte in the following solvents: N-octyl-2-pyrrolidone (NOP, Santa Cruz Biotechnology Inc.), acetonitrile (ACN, $99.8 \%$, SigmaAldrich), 1.2-dichlorobenzene (DCB, $99 \%$, Aldrich), 4-(3-phenylpropyl)-pyridine (PPP, $97 \%$, Aldrich), trifluorotoluene (TFT, $99 \%$, Acros Organics) and toluene (99.5\%, Aldrich). As fluorescence dye, we used rhodamine 101 inner salt purchased from Sigma-Aldrich which has relatively good solubility in a wide range of solvents.

\subsection{Electrochemical measurements}

The electrochemical measurements were performed using Autolab potentiostats (Metrohm Autolab) in a 
three-electrode set-up. All electrodes were sputtered $\mathrm{Au}$ microbands, on glass with a Ti adhesion layer, spanning the width of the microchannel patterned by standard photolithography. As working and pseudo-reference electrodes $100-\mu \mathrm{m}$-long gold bands were used, and the length of the counter electrode was $1000 \mu \mathrm{m}$. The gaps between the electrodes were $100 \mu \mathrm{m}$.

Cyclic voltammetry (CV) and square-wave voltammetry (SWV) of ferrocene were performed in each solvent with $1 \mathrm{~h}$ intervals for a total of $4 \mathrm{~h}$. As a measure of the compatibility of the solvent with the PDMS channel for electrochemical measurement, we used the stability of the current and SWV peak potential over the course of the experiments. The flow rate through the microchannel was $40 \mu \mathrm{min}^{-1}$ during measurements and kept at $1 \mu \mathrm{lmin}{ }^{-1}$ between the measurements, so as to not waste too much of the solvents and to provide fine time of solvent exposuring of PDMS channel. The flow was controlled using a Harvard Apparatus, Pump 11 elite syringe pump connected to the channel using polyethylene tubing.

Because we wanted to examine changes to the channels after the experiment series, the PDMS was not bonded to the glass supports, but instead held together by a PMMA support (Rozniecka et al. 2014).

To prevent the microchannel leakage, the whole PDMS block was hold by the pressure generated by the liquid flow introduced to microchannel in the withdraw mode. In the case of laser confocal imaging with TFT solvent, the PDMS was bonded to the glass plate by exposure to an $\mathrm{O}_{2}$ plasma for $50 \mathrm{~s}$ (Harrick, Model PDC-32G-2, $100 \mathrm{~W}$ ) providing contact between PDMS edges and glass.

\subsection{Swelling measurements}

The swelling was measured by immersing squares of PDMS in the various solvents and photographing them after 1, 5 and $24 \mathrm{~h}$ using a Canon EOD 5D digital camera. The distance between the parallel edges of the samples was measured from the photographs using the free software ImageJ. For accurate quantitative analysis, we measured five different samples for each solvent. The swelling parameter, $S$, was defined as the ratio of the final to the initial lengths of the sides of the samples.

\subsection{Fluorescence microscopy}

We performed laser scanning confocal images using a NIKON A1-R system. The system is based on a motorised NIKON TiE 2000 inverted microscope. The measurements were taken using a dry objective with $\times 10$ magnification (NA 0.3 , working distance $16 \mathrm{~mm}$ ). The liquid was stained with rhodamine 101 inner salt with a maximum of the absorption/emission at 560/589 $\mathrm{nm}$. The wavelength of the excitation diode laser was $561 \mathrm{~nm}$. We recorded the crosssection of the microfluidic channel using the 3D scanning mode of the confocal system. Separate focus planes were imaged at a frame rate of $1 / 15 \mathrm{fps}$. The distance between the focus planes was set to $9.645 \mu \mathrm{m}$. To increase the time resolution, we also imaged a single plane in the middle of the channel with a rate of $14.81 \mathrm{fps}$.

\section{Results and discussion}

The main goal of the present work was to investigate whether some solvents commonly thought of as incompatible with standard PDMS microchannels can be used for electrochemical studies on a relevant time scale. Since most electrochemical experiments are relatively fast, we used oxidation of ferrocene, a commonly used redox probe, as a model system and investigated how the electrochemical signal changes over the course of $4 \mathrm{~h}$.

In Whitesides' well-known study, the compatibility of organic solvents with PDMS was studied by looking at the swelling of a PDMS block after immersion in the solvent for $24 \mathrm{~h}$ (Lee et al. 2003). To be able to compare the results of our "electrochemical compatibility" with the swelling results published by the Whitesides group, we performed swelling experiments with the four liquids in our study not included in the original list of solvents. In Fig. 1, the bars show the swelling of PDMS in the various solvents after 1,5 and $24 \mathrm{~h}$. As we can see, significant swelling occurs only with TFT (close to $40 \%$ ) and to a smaller extent in DCB (about $10 \%$ ). In the inset of Fig. 1, the swelling ratio is plotted as a function of the Hildebrand solubility parameter, $\delta_{\mathrm{H}}$, which is defined as the square root of the cohesive energy density of the solvent (see e.g. Barton 1991 for more information on $\delta_{\mathrm{H}}$ ). This confirms the relationship established by Lee et al. (2003) that solvents with a solubility parameter close to that of PDMS $\left(7.3 \mathrm{cal}^{1 / 2} \mathrm{~cm}^{-3 / 2}\right)$ induce more swelling than those with a very different $\delta_{\mathrm{H}}$. The values of the solubility parameters for the solvents used in this study can be found in Table 1. For PPP and NOP, the values of the solubility parameter were estimated from similar compounds found in the literature. Most pyridines have $\delta_{\mathrm{H}}$ close to $10 \mathrm{cal}^{1 / 2} \mathrm{~cm}^{-3 / 2}$ (Abboud and Notari 1999), so we assumed the same value for PPP. For NOP, we used the value of the similar compound $\mathrm{N}$-methyl-2-pyrrolidone which is $11.1 \mathrm{cal}^{1 / 2} \mathrm{~cm}^{-3 / 2}$ (Abboud and Notari 1999). Still, we find that compounds with similar $\delta_{\mathrm{H}}$ can have very different swelling ratio. One explanation from the Whitesides paper (Lee et al. 2003) could be the influence of polar contribution to $\delta_{\mathrm{H}}$. However, in the case of DCB and PPP (both with $\delta_{\mathrm{H}} \approx 10 \mathrm{cal}^{1 / 2} \mathrm{~cm}^{-3 / 2}$ ), both molecules are quite polar. 


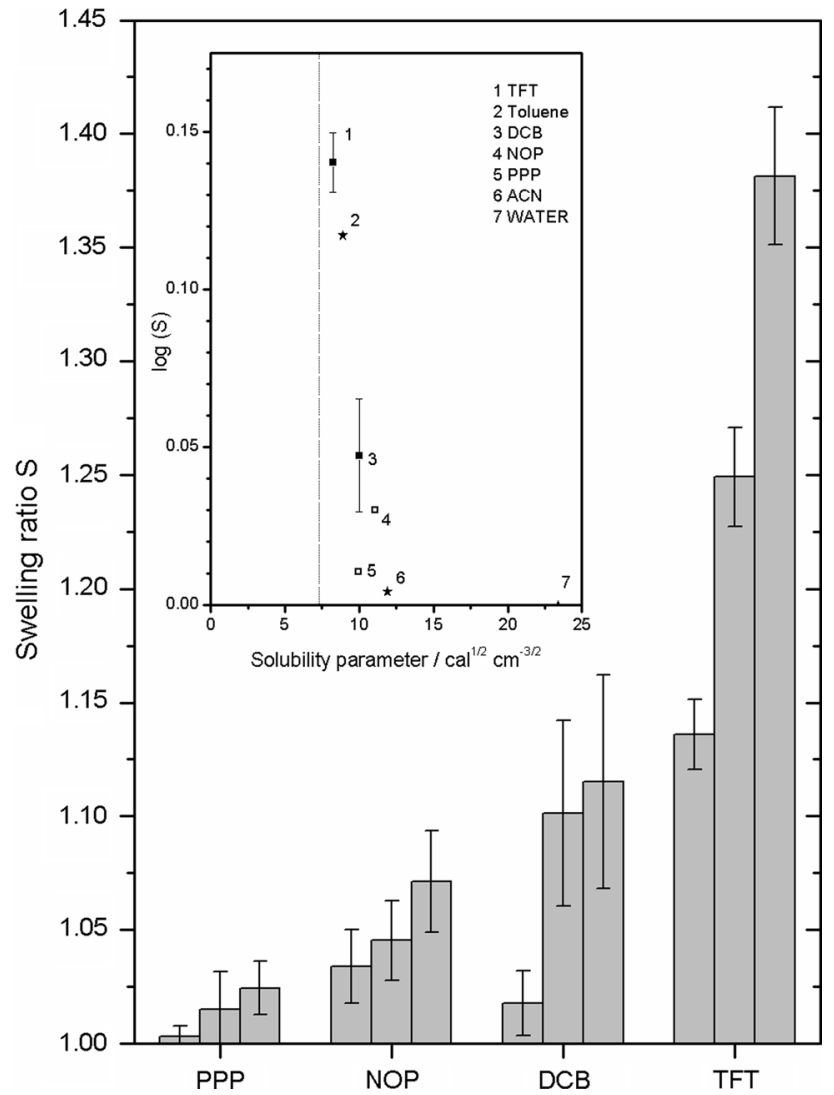

Fig. 1 Swelling ratio of PDMS blocks in various solvents measured after 1, 5 and $24 \mathrm{~h}$. Error bars represent standard deviation from 10 measurement + experimental precision. Inset Swelling ratio as a function of the solubility parameter. For open squares, $\delta_{\mathrm{H}}$ is an estimate (see text for more information). Stars represent data from ref (Lee et al. 2003)

Table 1 Values of solubility parameter, $\delta_{\mathrm{H}}$ and dipole moments, $\mu$ of the used solvents

\begin{tabular}{lll}
\hline Solvent & $\delta_{\mathrm{H}} / \mathrm{cal}^{1 / 2} \mathrm{~cm}^{-3 / 2}$ & $\mu / \mathrm{D}$ \\
\hline Trifluorotoluene & 8.26 & 2.9 \\
1,2-Dichlorobenzene & 10.02 & 2.16 \\
4-(3-Phenylpropyl)-pyridine & $\sim 10^{\mathrm{a}}$ & 2.9 \\
$N$-octyl-2-pyrrolidone & $\sim 11^{\mathrm{b}}$ & $-{ }^{\mathrm{c}}$ \\
Toluene & 8.9 & 0.4 \\
Acetonitrile & 11.9 & 4.0 \\
Water & 23.4 & 1.9 \\
\hline
\end{tabular}

Values of $\delta_{\mathrm{H}}$ come from ref (Abboud and Notari 1999). The values on the last three lines come from ref (Lee et al. 2003)

${ }^{a}$ Estimated value. Most pyridines have a listed value close to $10 \mathrm{cal}^{1 / 2} \mathrm{~cm}^{-3 / 2}$

b Estimated value. The similar compound $\mathrm{N}$-methyl-pyrrolidone has $\delta_{\mathrm{H}}=11.1 \mathrm{cal}^{1 / 2} \mathrm{~cm}^{-3 / 2}$

c Value not found
If swelling was indicative of compatibility with PDMS for electrochemical measurements, we would expect TFT and toluene to be particularly difficult with DCB and NOP on an intermediate level, and PPP and ACN to be un-problematic. Indeed, toluene is very hard to work with. We did not manage to record any useful electrochemical data using toluene because the solvent very quickly leaks out of the channel. For this reason, PDMS is often used as a membrane for pervaporative separation of toluene from water (Panek and Konieczny 2009).

However, with TFT as a solvent, it was possible to record stable electrochemical data for the whole $4 \mathrm{~h}$ duration of the experimental series. During the course of the experiment, the position of the oxidation peak in the square-wave voltammograms was very stable (Fig. 2a) and the current decreased only about $10 \%$ from its original value (Fig. 2b). This decrease is slightly higher than for the other compounds (Fig. 2c, d) and could be related to dissolution of PDMS oligomers that partially blocks the electrode (See Fig S1 \& S2 in ESI for CVs and SWVs of all compounds.). Although we could not see any deposition on the electrode after use, a significant decrease in the current when using other solvents was observed (see Fig S3 in ESI).

The electrochemical signals in PPP are very resistive, and the stability is hard to determine. However, the same behaviour is seen under static conditions (see Fig S4 in ESI) with the reference and working electrodes placed in close proximity. The use of PPP for electrochemical experiments is mainly restricted to ion transfer at three-phase junctions, in which case one of the liquid phases can be highly resistive without negative influence on the results (Katif et al. 2008; Collins et al. 2012).

Using TFT as a solvent, we observed a very fast partitioning of the fluorescent dye from the solvent into the PDMS (see Fig. 3 and Fig S5 in ESI). Using fluorescent laser scanning microscopy, we could follow the diffusion of the dye Rhodamine 101 inner salt into the PDMS. In Fig. 3, we see the fluorescence signal along a line perpendicular to the channel over time. The point where the TFT/Rhodamine is pumped into the channel can clearly be seen, and then the fluorescent signal is spreading away from the channel. By fitting the fluorescence as a function of space and/or time, we can determine the diffusion coefficient of Rhodamine in TFT into PDMS. To a first approximation, we can see this as one-dimensional diffusion from a point source with a constant concentration, $c_{0}$. For such a system, the diffusion equation can be solved analytically as

$c(x, t)=c_{0} \operatorname{erfc}\left(\frac{x}{2 \sqrt{D t}}\right)$ 
Fig. 2 Square-wave voltammograms (a) and cyclic voltammograms (b) of the oxidation of ferrocene in TFT after 0 (black), 1 (red), 2 (green), 3 (blue) and $4 \mathrm{~h}$ (magenta). c Change in position of the ferrocene oxidation peak over the course of the experiments for the different solvents. d Limiting current in $\mathrm{CVs}$ of ferrocene oxidation in different solvents. Current normalised to the first measurement in the time series. Black squares-ACN, red circles$\mathrm{DCB}$, blue triangles-NOP, pink diamonds-TFT, olive open triangles - PPP and brown stars-water
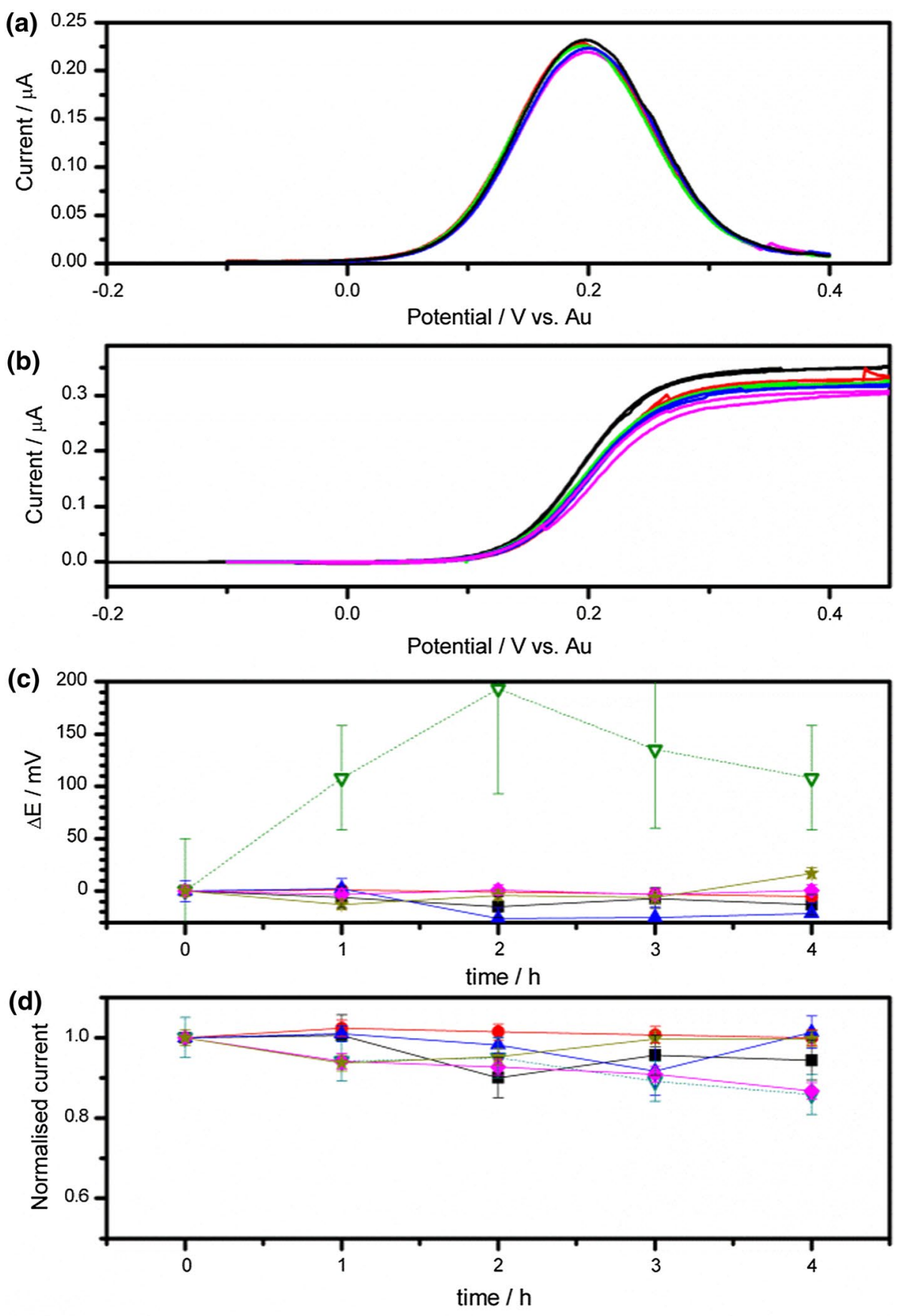

Fitting Eq. 1 to fluorescence profiles at a fixed $t$ or fixed $x$ (see supplemental information), we get a diffusion coefficient $D=6-12 \times 10^{-8} \mathrm{~cm}^{2} \mathrm{~s}^{-1}$, where the uncertainty comes both from the one-dimensional approximation (the 2D system does not have an analytical solution) and from the bleaching of the fluorophore which makes the fluorescent profile dependent not only on diffusion.
Despite this, the obtained value is consistent with values in the literature for diffusion of toluene and benzene into PDMS $\left(2 \times 10^{-8}\right.$ and $7 \times 10^{-8} \mathrm{~cm}^{2} \mathrm{~s}^{-1}$, respectively) (Chao et al. 2011), which shows that the Rhodamine is carried with the solvent almost unhindered through the PDMS. This might be a problem if analyte is lost into the PDMS slab. 


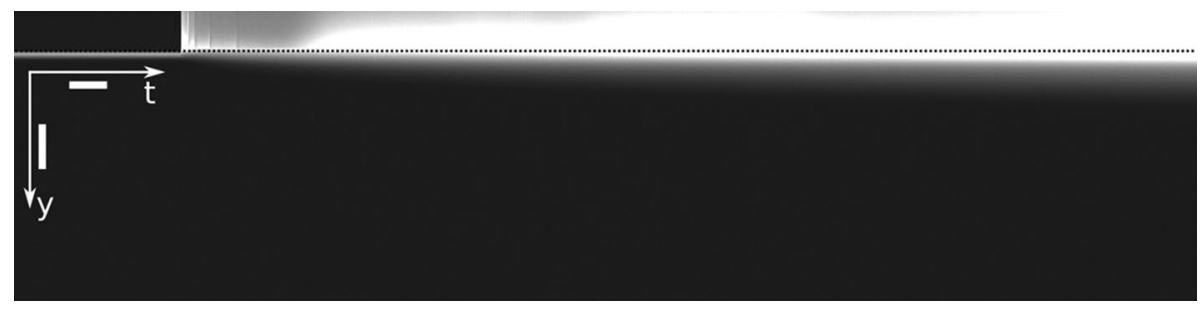

Fig. 3 Fluorescence from Rhodamine 101 inner salt imaged by laser scanning confocal microscopy. The image shows the fluorescence along a single horizontal line over time. The channel can be seen at the top of the images outlined by scattering from the channel wall (marked by a dashed line), and the lower part of the image is inside

\section{Conclusions}

The electrochemical measurements show that many organic solvents thought of as incompatible with microchannels made from PDMS actually provide stable voltammetric signals over the course of several hours. Although the solvent with the highest swelling parameter, toluene, is not compatible with PDMS, trifluorotoluene, which shows almost as high swelling as toluene, gives relatively stable electrochemistry in a PDMS channel. The other tested solvents, ranging from moderate to low swelling, showed very consistent electrochemistry over the whole experimental series, the exception being PPP which shows very resistive electrochemistry also under static conditions.

The conclusion of this study, in contrast to the paper by Lee et al. (2003), is that although there is a good correlation between swelling and solubility parameter, we do not find that swelling is a good measure for compatibility with PDMS in electrochemical experiments.

Acknowledgments The authors thank Dr Tomasz Kalwarczyk from the Institute of Physical Chemistry for his help with confocal microscopy. This work was financially supported by the Polish National Science Centre through Grants NCN 2014/15/D/ST4/03003 (WA), NCN 2015/16/T/ST4/00495 (DK), and NCN 2014/15/B/ST4/04646 (MJN).

Open Access This article is distributed under the terms of the Creative Commons Attribution 4.0 International License (http://creativecommons.org/licenses/by/4.0/), which permits unrestricted use, distribution, and reproduction in any medium, provided you give appropriate credit to the original author(s) and the source, provide a link to the Creative Commons license, and indicate if changes were made.

\section{References}

Abboud J-LM, Notari R (1999) Critical compilation of scales of solvent parameters. Part I. Pure, non-hydrogen bond donor solvents. Pure Appl Chem 71:645-718. doi:10.1351/pac199971040645

Adamiak W, Shul G, Rozniecka E, Satoh M, Chen J, Opallo M (2011) Voltammetry of $\mathrm{Mn}(\mathrm{III})$ Porphyrin in the PDMS slab. The height of the line is in the middle of the channel. The moment when the dye is pumped into the channel can clearly be seen as the start of fluorescence in the channel. The flow rate is kept at $10 \mu \mathrm{min}^{-1}$ during the experiment. Scale bars are $10 \mathrm{~s}$ in $t$ and $200 \mu \mathrm{m}$ in $y$

Trihexyl(tetradecyl)-phosphonium Tris(pentafluoroethyl)trifluorophosphate supported toluene in contact with an aqueous electrolyte. Electroanalysis 23:1921-1927. doi:10.1002/ elan.201100188

Barton AFM (1991) CRC handbook of solubility parameters and other cohesion parameters, 2nd edn. CRC Press, Boca Raton

Cate DM, Adkins JA, Mettakoonpitak J, Henry CS (2015) Recent developments in paper-based micro fluidic devices. Anal Chem. doi:10.1021/ac503968p

Chao K-P, Wang V-S, Yang H-W, Wang C-I (2011) Estimation of effective diffusion coefficients for benzene and toluene in PDMS for direct solid phase microextraction. Polym Test 30:501-508. doi:10.1016/j.polymertesting.2011.04.004

Collins AM, Blanchard GJ, Marken F (2012) Spectroelectrochemical investigation of TPPMn(III/II)-driven liquid I liquid I electrode triple phase boundary anion transfer into 4-(3-Phenylpropyl)Pyridine: $\mathrm{ClO} 4-, \mathrm{CO} 3 \mathrm{H}-, \mathrm{Cl}-$, and F-. Electroanalysis 24:246253. doi:10.1002/elan.201100623

Duffy NW, Bond AM (2006) Macroelectrode voltammetry in toluene using a phosphonium-phosphate ionic liquid as the supporting electrolyte. Electrochem Commun 8:892-898. doi:10.1016/j. elecom.2006.03.036

Dumitrescu I, Yancey DF, Crooks RM (2012) Dual-electrode microfluidic cell for characterizing electrocatalysts. Lab Chip 12:986. doi:10.1039/c2lc21181e

El-Ali J, Sorger PK, Jensen KF (2006) Cells on chips. Nature 442:403-411. doi:10.1038/nature05063

Ghaemmaghami AM, Hancock MJ, Harrington H, Kaji H, Khademhosseini A (2012) Biomimetic tissues on a chip for drug discovery. Drug Discov Today 17:173-181. doi:10.1016/j. drudis.2011.10.029

Han Z, Li W, Huang Y, Zheng B (2009) Measuring rapid enzymatic kinetics by electrochemical method in droplet-based microfluidic devices with pneumatic valves. Anal Chem 81:5840-5845. doi:10.1021/ac900811y

Jedraszko J, Nogala W, Adamiak W, Rozniecka E, Lubarska-Radziejewska I, Girault HH, Opallo M (2013) Hydrogen peroxide generation at liquidlliquid interface under conditions unfavorable for proton transfer from aqueous to organic phase. J Phys Chem C 117:20681-20688. doi:10.1021/jp406422d

Kaluza D, Adamiak W, Kalwarczyk T, Sozanski K, Opallo M, JönssonNiedziolka M (2013) Anomalous effect of flow rate on the electrochemical behavior at a liquidlliquid interface under microfluidic conditions. Langmuir 29:16034-16039. doi:10.1021/la403614z

Kaluza D, Adamiak W, Opallo M, Jonsson-Niedziolka M (2014) Comparison of ion transfer thermodynamics at microfluidic and droplet-based three phase electrodes. Electrochim Acta 132:158 164. doi:10.1016/j.electacta.2014.03.105 
Katif N, MacDonald SM, Kelly AM, Galbraith E, James TD, Lubben AT, Opallo M, Marken F (2008) Electrocatalytic determination of sulfite at immobilized microdroplet liquidlliquid interfaces: the EIC $^{\prime}$ mechanism. Electroanalysis 20:469-475. doi:10.1002/ elan. 200704127

Kim HS, Devarenne TP, Han A (2015) A high-throughput microfluidic single-cell screening platform capable of selective cell extraction. Lab Chip. doi:10.1039/C4LC01316F

Kjeang E, Djilali N, Sinton D (2009) Microfluidic fuel cells: a review. J Power Sources 186:353-369. doi:10.1016/j.jpowsour.2008.10.011

Kowski M, Stojek Z, Palys MJ (2009) Significance of comproportionation reaction in multi-step electrochemical reduction of fullerene C60. Electrochem Commun 11:905-908. doi:10.1016/j. elecom.2009.02.024

Lee JN, Park C, Whitesides GM (2003) Solvent compatibility of poly(dimethylsiloxane)-based microfluidic devices. Anal Chem 75:6544-6554. doi:10.1021/ac0346712

MacDonald SM, Watkins JD, Gu Y, Yunus K, Fisher AC, Shul G, Opallo M, Marken F (2007) Electrochemical processes at a flowing organic solventlaqueous electrolyte phase boundary. Electrochem Commun 9:2105-2110. doi:10.1016/j.elecom.2007.05.031

MacDonald SM, Watkins JD, Bull SD, Davies IR, Gu Y, Yunus K, Fisher AC, Bulman Page PC, Chan Y, Elliott C, Marken F (2008) Two-phase flow electrosynthesis: comparing N-octyl-2-pyrrolidone-aqueous and acetonitrile-aqueous three-phase boundary reactions. J Phys Org Chem 22:52-58. doi:10.1002/poc.1424

Martinez AW, Phillips ST, Butte MJ, Whitesides GM (2007) Patterned paper as a platform for inexpensive, low-volume, portable bioassays. Angew Chemie Int Ed 46:1318-1320. doi:10.1002/ anie. 200603817

Martinez AW, Phillips ST, Carrilho E, Thomas SW, Sindi H, Whitesides GM (2008) Simple telemedicine for developing regions: camera phones and paper-based microfluidic devices for real-time, off-site diagnosis. Anal Chem 80:3699-3707. doi:10.1021/ac800112r

McDonald JC, Whitesides GM (2002) Poly(dimethylsiloxane) as a material for fabricating microfluidic devices. Acc Chem Res 35:491-499. doi:10.1021/ar010110q

Mu X, Zheng W, Sun J, Zhang W, Jiang X (2013) Microfluidics for manipulating cells. Small 9:9-21

Olaya AJ, Ge P, Girault HH (2012) Ion transfer across the waterltrifluorotoluene interface. Electrochem Commun 19:101104. doi:10.1016/j.elecom.2012.03.010
Panek D, Konieczny K (2009) Pervaporative separation of toluene from wastewaters by use of filled and unfilled poly(dimethylosiloxane) (PDMS) membranes. Desalination 241:197-200. doi:10.1016/j.desal.2007.11.085

Pumera M, Merkoçi A, Alegret S (2006) New materials for electrochemical sensing VII. Microfluidic chip platforms. TrAC Trends Anal Chem 25:219-235. doi:10.1016/j.trac.2005.08.005

Ren K, Zhou J, Wu H (2013) Materials for microfluidic chip fabrication. Acc Chem Res 46:2396-2406. doi:10.1021/ar300314s

Reyes DR, Iossifidis D, Auroux P-A, Manz A (2002) Micro total analysis systems. 1. Introduction, theory, and technology. Anal Chem 74:2623-2636. doi:10.1021/ac0202435

Rozniecka E, Jonsson-Niedziolka M, Celebanska A, Niedziolka-Jonsson J, Opallo M (2014) Selective electrochemical detection of dopamine in a microfluidic channel on carbon nanoparticulate electrodes. Analyst 139:2896-2903. doi:10.1039/c3an02207b

Shim J-U, Cristobal G, Link DR, Thorsen T, Jia Y, Piattelli K, Fraden S (2007) Control and measurement of the phase behavior of aqueous solutions using microfluidics. J Am Chem Soc 129:8825-8835. doi:10.1021/ja071820f

Stalder R, Roth GP (2013) Preparative microfluidic electrosynthesis of drug metabolites. ACS Med Chem Lett 4:1119-1123. doi:10.1021/ml400316p

Strutwolf J, Collins CJ, Adamiak W, Arrigan DWM (2010) Potentiometric investigation of protonation reactions at aqueous-aqueous boundaries within a dual-stream microfluidic structure. Langmuir 26:18526-18533. doi:10.1021/la102149c

Swensen JS, Xiao Y, Ferguson BS, Lubin AA, Lai RY, Heeger AJ, Plaxco KW, Soh HT (2009) Continuous, real-time monitoring of cocaine in undiluted blood serum via a microfluidic, electrochemical aptamer-based sensor. J Am Chem Soc 131:42624266. doi:10.1021/ja806531z

Unger MA, Chou H-P, Thorsen T, Scherer A, Quake SR (2000) Monolithic microfabricated valves and pumps by multilayer soft lithography. Science 288:113-116

Whitesides GM (2002) The origins and the future of microfluidics. Nature 442:368-373

Wu H, Odom TW, Chiu DT, Whitesides GM (2003) Fabrication of complex three-dimensional microchannel systems in PDMS. J Am Chem Soc 125:554-559. doi:10.1021/ja021045y 